\title{
Epidemiologia das helmintoses gastrintestinais de ovinos no Planalto Catarinense
}

\author{
Epidemiology of sheep gastrintestinal helminthosis in "Planalto Catarinense” Region, Brazil
}

\author{
César Itaqui Ramos ${ }^{1}$ Valdomiro Bellato ${ }^{2}$ Antonio Pereira de Souza ${ }^{2}$ Volney Silveira de Avila \\ Guilherme Caldeira Coutinho $^{1}$ Celso Augustinho Dalagnol ${ }^{1}$
}

\section{RESUMO}

\begin{abstract}
Este trabalho foi desenvolvido em três propriedades rurais nos municípios de Lages, São Joaquim e Campos Novos, estado de Santa Catarina, com os objetivos de determinar a prevalência, a intensidade e a variação sazonal de helmintos gastrintestinais e pulmonares em ovinos no Planalto Catarinense. Com base nos resultados aintenção é propor um esquema estratégico de controle. Para isso, foram utilizados mensalmente três cordeiros traçadores por propriedade, os quais, antes de serem conduzidos às mesmas, foram estabulados por 30 dias e executados tratamentos supressivos com anti-helmínticos de diferentes princípios ativos, com exames parasitológicos semanais para verificar a total eliminação de infecção parasitária. A seguir, foram encaminhados às três propriedades onde permaneceram em pastejo por 28 dias, sendo posteriormente recolhidos ao estábulo por mais 20 dias. Após foram sacrificados e realizada a coleta de alíquotas de $10 \%$ dos conteúdos do abomaso e intestino delgado, todos os helmintos do intestino grosso e pulmão. As maiores infecções por Haemonchus contortus ocorreram durante o período de outubro a março. O parasitismo por Trichostrongylus axei $e$ Trichostrongylus colubriformis teve índices crescentes ao longo dos anos experimentais, apresentando maior pico de maio a outubro de 1999. Predominaram as espécies, no abomaso: Haemonchus contortus (100\%); Trichostrongylus axei (98,7\%); Trichostrongylus colubriformis (1,3\%); Teladorsagia circumcincta (100\%); Ostertagia ostertagi (100\%). No intestino delgado: Trichostrongylus colubriformis (100\%); Cooperia punctata (69,1\%); Cooperia pectinata (18,4\%); Cooperia curticei (6,9\%); Cooperia oncophora (4,8\%); Cooperia spatulata $(0,8 \%)$ e Nematodirus spathiger (100\%). No intestino grosso: Oesophagostomum venulosum (100\%) e Trichuris ovis (100\%).

No pulmão, não foram encontrados parasitos.
\end{abstract}

Palavras-chave: ovinos, epidemiologia, helmintos.

\section{ABSTRACT}

This work was developed for proposing a control strategy of sheep helminthes in the Santa Catarina Plateau. The prevalence, intensity and seasonal variation of gastrointestinal and pulmonary helminths in three farms of Lages, São Joaquim, and Campos Novos in Santa Catarina State, Brazil were assessed. Monthly, three tracer lambs were used by property. They had been previously housed for 30 days and suppressively treated by anthelmintics with different active ingredients followed by weekly parasitological examinations to assure the total elimination of parasitic infection. After this, they were taken back to their respective farms, where they grazed for 28 days and housed again for another 20 days. After this period, they were sacrificed for estimating the total worm burden, and $10 \%$ of abomasum and small intestine material was sampled as well as all the helminthes from the large intestine and the lungs. The heaviest Haemonchus contortus infection was registered from October to March. Trichostrongylus colubriformis and Trichostrongylus axei had increased rates through the experimental years, showing the peak from May to October of 1999. The most prevailing species in the abomasum were $\boldsymbol{H}$. contortus (100\%), T. axei (98.7\%), T. colubriformis (1.3\%), Teladorsagia circumcincta (100\%) and Ostertagia ostertagi (100\%); in the small intestine: T. colubriformis (100\%), Cooperia punctata (69.1\%), C. pectinata (18.4\%), C. curticei (6.9\%), C. oncophora (4.8\%), C. spatulata (0.8\%) and Nematodirus spathiger (100\%). In the large intestine: Oesophagostomum venulosum (100\%) and Trichuris ovis $(100 \%)$. No worms were detected in the lungs.

Key words: sheep, epidemiology, helminths.

\footnotetext{
${ }^{1}$ Médico Veterinário, Pesquisador, Mestre, Estação Experimental de Lages, Epagri, CP 181, 88502-970, Lages, SC. E-mail: itaqui@epagri.rct-sc.br.

${ }^{2}$ Médico Veterinário, Pesquisador, PhD, Departamento de Medicina Veterinária Preventiva e Tecnologia, Centro de Ciências Agroveterinárias (CAV), Universidade do Estado de Santa Catarina (UDESC).
} 
INTRODUÇÃo

O Brasil, com sua enorme extensão territorial e clima favorável à espécie ovina, apresenta altíssimo potencial para tornar-se importante produtor mundial de ovinos. Entretanto, o produtor rural brasileiro ainda não foi devidamente conscientizado a respeito desta potencialidade (SIQUEIRA, 1999).

O estado de Santa Catarina possui um rebanho ovino estimado em 202.694 cabeças, com características voltadas principalmente para a produção de carnes e o sistema de criação é intensivo ou semi-intensivo. Este rebanho está distribuído, praticamente, em todos os municípios, com predominância na Região Serrana (38,70\%) e Oeste Catarinense (32,79\%) (IBGE, 1997). Estas regiões compreendem o Planalto Catarinense, cujo clima predominante é considerado, segundo a classificação de Köppen, temperado quente e chuvoso, (Cfb), com verão mais frio, em relação ao Litoral (Cfa), (RITTER \& SORRENSON, 19885).

Um dos principais problemas encontrados na ovinocultura, e que limita consideravelmente o aproveitamento econômico destes animais, são as parasitoses gastrintestinais. Os ovinos são parasitados por helmintos em todas as faixas etárias e a sua ação negativa não acontece apenas no atraso de desenvolvimento corporal dos cordeiros, mas também na produção e qualidade da carne e da lã (PINHEIRO, 1979). Observações em lotes de ovinos tratados a cada quinze dias e a cada mês, tiveram melhores pesos vivos e maior produção de lã. A percentagem média de lã de melhor qualidade foi de aproximadamente $70 \%$. A percentagem de mortes por verminose foi em média de $40 \%$ do grupo não tratado e inexistente nos animais medicados duas vezes ao mês ou mensalmente. Neste trabalho, ficou plenamente evidenciado que os ovinos jovens, especialmente no primeiro ano de vida, devem ser submetidos a um controle adequado dos parasitos internos, para que suas produções futuras não sejam permanentemente prejudicadas (PINHEIRO, 1982).

Algumas práticas contribuem para reduzir a verminose, dentre elas, alinham-se a manutenção de bom estado corporal, a separação por idade, e a lotação adequada nos pastos (RODA et al., 1986).

Os trabalhos de GORDON (1948), na Austrália, sobre a epidemiologia da helmintose ovina tiveram fundamental influência na metodologia aplicada nos estudos que posteriormente foram efetuados no Rio Grande do Sul e em outras regiões do Brasil.
No Brasil, GONÇALVES (1974) realizou o primeiro trabalho em epidemiologia da helmintose ovina no município de Guaíba (RS) durante os anos de 1961 a 1965. Posteriormente, SANTIAGO et al. (1976) relataram pesquisas em epidemiologia e controle da helmintose ovina em Itaqui (RS). Nos trabalhos acima citados, os autores trabalharam com epidemiologia de rebanho, isto é, os cordeiros que eram necropsiados permaneciam sempre juntos com o rebanho antes de serem abatidos. Ainda no Rio Grande do Sul, no município de Bagé, durante os anos de 1976 a 1978, PINHEIRO et al. (1987) desenvolveram estudos epidemiológicos utilizando a técnica com cordeiros traçadores.

Em Santa Catarina, na região dos Campos de Lages, RAMOS et al. (1985), através de coletas de amostras de conteúdos gastrintestinais de ovinos, determinaram a prevalência dos principais gêneros e espécies de helmintos, destacando-se como principais, Haemonchus contortus (61,3\%), Trichostrongylus axei (54,8\%), Ostertagia (Teladorsagia) circumcincta (25,8\%), Trichostrongylus colubriformis $(48,2 \%)$, Trichostrongylus longispicularis (25,8\%), Oesophagostomum columbianum (38,7\%), Oesophagostomum venulosum e Trichuris ovis (32,3\%) e Muellerius spp. (19,4\%). Dando continuidade a estes trabalhos em Santa Catarina, SOUZA et al. (2000) determinaram o período necessário de descanso de pasto para ocorrer desinfestação por larvas de nematódeos gastrintestinais de ovinos em campos naturais, verificando que, na primavera, são necessários 42 a 56 dias para ocorrer uma redução apreciável destas larvas nas pastagens para a maioria dos gêneros, aumentando para 70 a 84 dias se for no verão.

Pesquisa em epidemiologia das helmintoses gastrintestinais nos ovinos é a parcela que falta para que se possa associar aos demais conhecimentos e estabelecer formas adequadas de controle estratégico visando à melhoria da produtividade e ao desenvolvimento econômico da ovinocultura catarinense.

\section{MATERIAL E MÉTODOS}

Mensalmente, durante outubro de 1997 a setembro de 2001, nove ovinos, foram estabulados durante 30 dias para realização de tratamentos supressivos com anti-helmínticos de diferentes princípios ativos, com poder residual não superior ao período de estabulação. Durante esse período, os animais receberam água e alimentos livres de contaminação por larvas de helmintos. Semanalmente, foram coletadas e examinadas amostras de fezes para comprovar a total desinfecção. Logo após esta fase, estes animais, chamados traçadores 
foram conduzidos, no início de cada mês, três para cada uma das propriedades rurais representativas da região, com criação de ovinos junto com os bovinos nos municípios de Lages (27 48'05” S, 50²0’06” W e

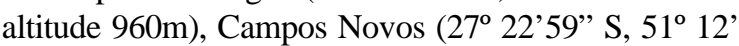
$55^{\prime \prime}$ W e altitude $964 \mathrm{~m}$ ) e São Joaquim ( $28^{\circ} 17^{\prime} 39^{\prime \prime} \mathrm{S}$, $49^{\circ} 55^{\prime} 56^{\prime}$ ' W e altitude $1415 \mathrm{~m}$ ), onde permaneceram por 28 dias em pastejo junto com os demais ovinos das propriedades. Posteriormente, repetindo-se a mesma metodologia, retornaram ao estábulo por mais 20 dias antes de serem abatidos, tempo suficiente para o desenvolvimento das infecções por larvas dos helmintos gastrintestinais, conforme metodologia utilizada por PINHEIRO et al. (1987).

De acordo com FREITAS \& COSTA (1970), nas necropsias dos animais, executou-se coleta total dos helmintos do intestino grosso e pulmão, coleta de alíquotas de $10 \%$ dos conteúdos do abomaso e intestino delgado, as quais foram em seguida, fixadas pela técnica de REINECKE (1968).

A identificação dos gêneros de helmintos recuperados foi realizada com auxílio do estereomicroscópio, segundo UENO \& GONÇALVES (1998), sendo separados do total até 100 helmintos por gênero, para posterior identificação de espécie, com base nas características morfológicas dos espículos, segundo SOULSBY (1987).

\section{RESULTADOS E DISCUSSÃO}

A prevalência das espécies dos helmintos gastrintestinais de ovinos, durante os quatro anos experimentais, foi a seguinte: no abomaso - $\boldsymbol{H}$. contortus (100\%); T. axei (98,7\%); T. colubriformis (1,3\%)); T. circumcincta (100\%); O. ostertagi (100\%). No intestino delgado - T. colubriformis (100\%); C. punctata (69,1\%); C. pectinata $(18,4 \%)$; C. curticei (6,9\%); C. oncophora (4,8\%); C. spatulata $(0,8 \%)$ e $N$. spathiger (100\%). No intestino grosso O. venulosum (100\%) e Trichuris ovis (100\%). Estes resultados confirmam as mesmas tendências quanto às principais espécies identificadas por RAMOS et al. (1985) nesta mesma região. Registra-se como alteração, a ausência total de helmintos pulmonares. Este fato pode estar relacionado com o uso intensivo das avermectinas no controle das helmintoses nos rebanhos de ovinos desde a década de 80 .

Convém ressaltar que as cargas parasitárias dos ovinos abatidos referem-se a uma infecção de apenas 28 dias, tempo de permanência no campo, diferente do que acontece normalmente no rebanho às quais são acumulativas. Tendo em vista que os resultados obtidos nos três municípios seguiram, durante os quatro anos experimentais, uma mesma tendência nas flutuações estacionais quanto às intensidades de infecção parasitárias, optou-se pela análise das médias das três propriedades.

A flutuação média estacional das infecções por helmintos localizadas no abomaso dos ovinos traçadores, no período de outubro de 1997 a setembro de 2001, nos municípios de Lages, São Joaquim e Campos Novos, estão apresentadas na figura 1.

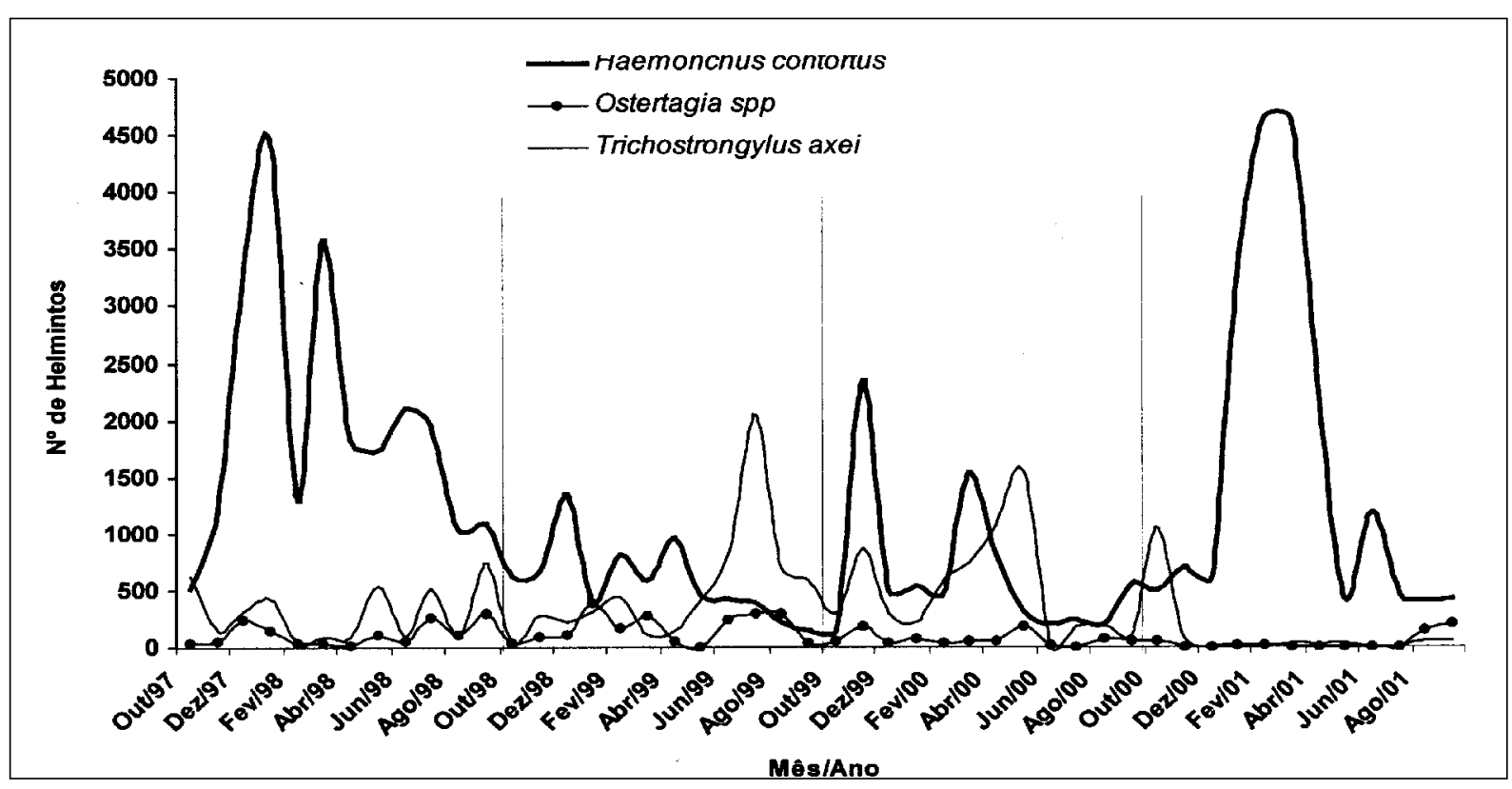

Figura 1 - Flutuação estacional de helmintos do abomaso de ovinos, no Planalto Catarinense, no período de outubro de 1997 a setembro de 2001.

Ciência Rural, v.34, n.6, nov-dez, 2004. 
Quanto ao gênero Haemonchus, identificou-se apenas a espécie $\boldsymbol{H}$. contortus, a qual se apresentou com as mais altas intensidades médias de infecção, variando de um modo geral entre de 500 a 4500 exemplares. Considerando a sua elevada prevalência e a alta patogenicidade, segundo UENO \& GONÇALVES (1998), uma infecção com 500 Haemonchus spp., é igual a uma unidade de carga patogênica, verifica-se que, na maioria dos meses, o número deste gênero foi superior a 500, o que permite afirmar que, no Planalto Catarinense, foi o principal parasito do abomaso dos ovinos. Os maiores picos de infecções verificadas com $\boldsymbol{H}$. contortus ocorreram durante os meses de verão e outono. Resultados semelhantes foram descritos por GONÇALVES (1974), SANTIAGO et al. (1976) e PINHEIRO et al. (1987), no Rio Grande do Sul, quando efetuaram os primeiros estudos epidemiológicos com ovinos em pastoreio permanente. Ambos concluíram que $\boldsymbol{H}$. contortus foi o helminto com maior freqüência ao longo dos meses de verão e outono. Possivelmente, a indicação de tratamentos estratégicos na primavera evitaria a contaminação das pastagens, reduzindo as infecções no verão e outono. Quanto ao T. axei, teve índices crescentes a partir do outono e inverno, principalmente no segundo e terceiro ano das avaliações, ultrapassando em alguns meses, aos índices observados para a espécie $\boldsymbol{H}$. contortus. Os animais necropsiados apresentaram, durante os quatro anos, baixos níveis de infecções por $\boldsymbol{T}$. circumcincta e Ostertagia spp., não sendo considerada como importante sua análise individual.

Quanto à flutuação estacional dos principais helmintos, em relação aos dados climáticos, observase que a precipitação pluviométrica (Figura 2) mais intensa no primeiro ano (1997/98) e no quarto ano (2000/01), provavelmente foi a principal causa das maiores intensidades de infecções por $\boldsymbol{H}$. contortus neste período. Em contrapartida, nos dois anos intermediários (1998/99 e 1999/00), ocorreram maiores cargas de $\mathbf{T}$. axei principalmente no outono e inverno, períodos que apresentaram uma moderada antecipação das temperaturas médias abaixo de $15^{\circ} \mathrm{C}$ a partir do mês de abril, dificultando novas infecções por $\boldsymbol{H}$. contortus. A menor concorrência entre estas duas espécies facilitou ao T. axei maiores infecções. GORDON (1948) considerou que as condições ecológicas ideais para o desenvolvimento de surtos de hemoncose (H. contortus) seria com uma precipitação mensal de $50 \mathrm{~mm}$ e uma temperatura média mensal acima de $17,7^{\circ} \mathrm{C}$. Da mesma forma, LEVINE et al. (1974) verificaram que $50 \mathrm{~mm}$ de precipitação e 15 a $37^{\circ} \mathrm{C}$ de temperatura média mensal indicavam ótimas condições para a transmissão de $\boldsymbol{H}$. contortus, e que o potencial de transmissão aumenta nos períodos estacionais com temperaturas mais amenas, o que esclarece as altas contaminações de outono.

Com relação ao gênero Trichostrongylus, LEVINE (1963) descreve que este é mais resistente na fase larval do que o gênero Oesophagostomum e Haemonchus, o que justifica as cargas mais altas nas condições menos favoráveis de precipitação pluviométrica e temperaturas inferiores a $15^{\circ} \mathrm{C}$. REINECKE (1970) também constatou na África do Sul, que bastava 5 a $10 \mathrm{~mm}$ de precipitação para estimular o desenvolvimento de Trichostrongylus spp.

RAMOS \& PALOSCHI (1986), trabalhando com a epidemiologia das helmintoses dos bovinos de corte na mesma região, constataram semelhança com a epidemiologia dos helmintos de ovinos com relação ao Trichostrongylus spp. e ao Haemonchus spp., fato este que pode estar associado ao sistema de manejo nas propriedades e à possibilidade de infecção cruzada destes helmintos, quando as duas espécies animais estão sendo manejadas nas mesmas áreas.

Dentre os parasitas do intestino delgado, a espécie que ocorreu com maior intensidade foi o T. colubriformis, principalmente nos meses de verão, outono e inverno, variando as intensidades de infecção de 1000 a 2000 espécimes. Observouse que, nos dois anos intermediários (1998/99 e 1999/00), repetiram-se as mesmas tendências de maiores cargas de $\boldsymbol{T}$. colubriformis, de maio a setembro, contrário ao $\boldsymbol{H}$. contortus, corroborando as mesmas tendências anteriormente discutidas em outras regiões com relação ao T. axei e os dados climáticos (LEVINE, 1963; REINECKE, 1970). Possivelmente, a indicação de tratamentos estratégicos em março e abril evitaria a contaminação das pastagens, reduzindo as altas infecções destas espécies a partir de maio. No entanto, as hipóteses de tratamentos estratégicos para os principais gêneros de helmintos de ovinos, nesta região, devem ser aviliadas através de estudos experimentais.

N. spathiger e Cooperia spp., (principalmente $\boldsymbol{C}$. punctata e $\boldsymbol{C}$. pectinata) estavam sempre presentes nas necropsias, mas com baixas infecções, evidenciando pouca importância no contexto epidemiológico (Figura 3). Em relação aos helmintos do intestino grosso, tanto $\boldsymbol{O}$. venulosum como T. ovis, foram encontrados em baixos índices, com predominância destas duas espécies no verão e outono, (Figura 4). Baixas infecções por $\boldsymbol{O}$. 


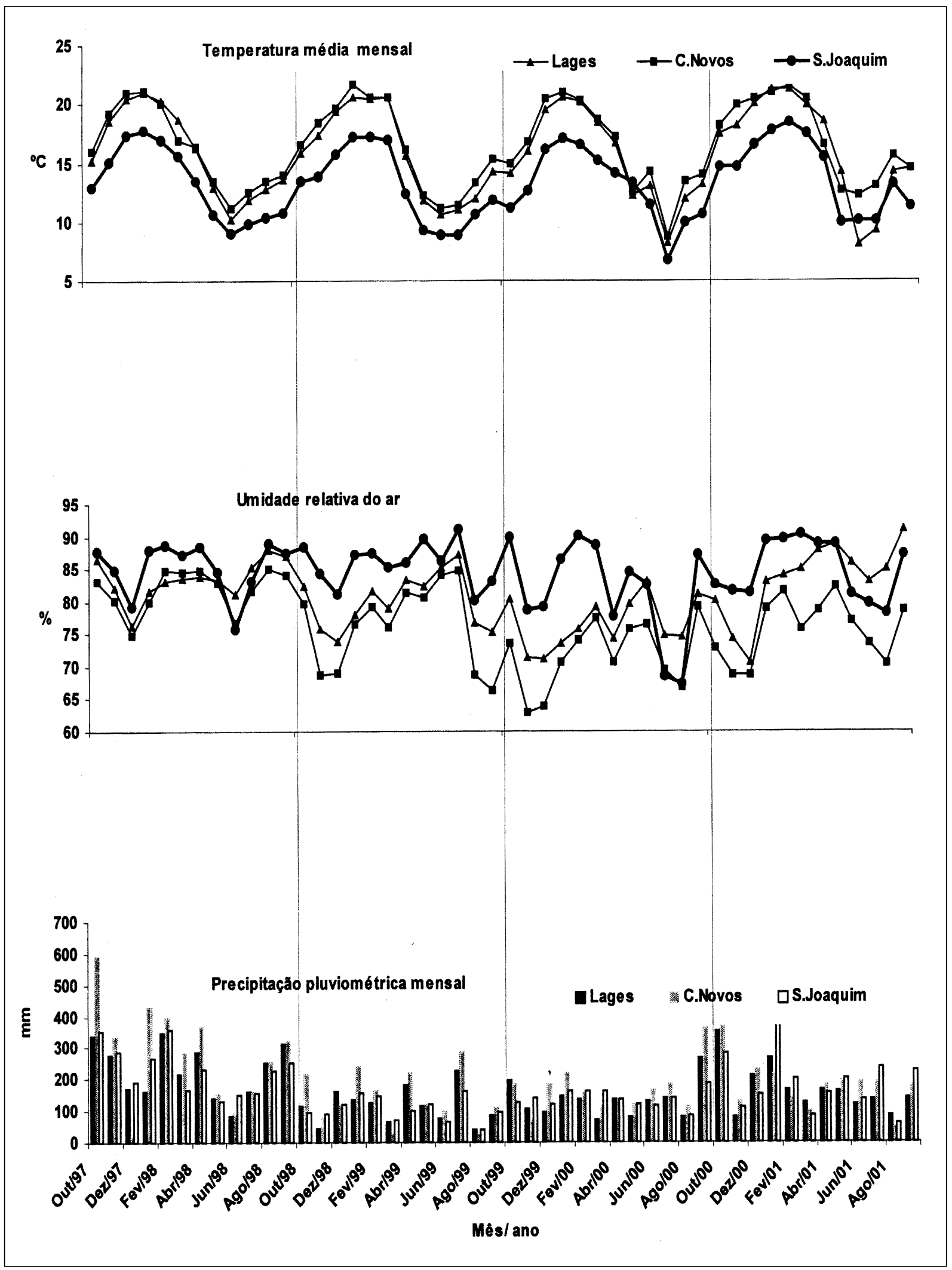

Figura 2 - Dados de clima no período de outubro de 1997 a setembro de 2001, nos municípios de Lages, Campos Novos e São Joaquim, SC.

Ciência Rural, v.34, n.6, nov-dez, 2004. 


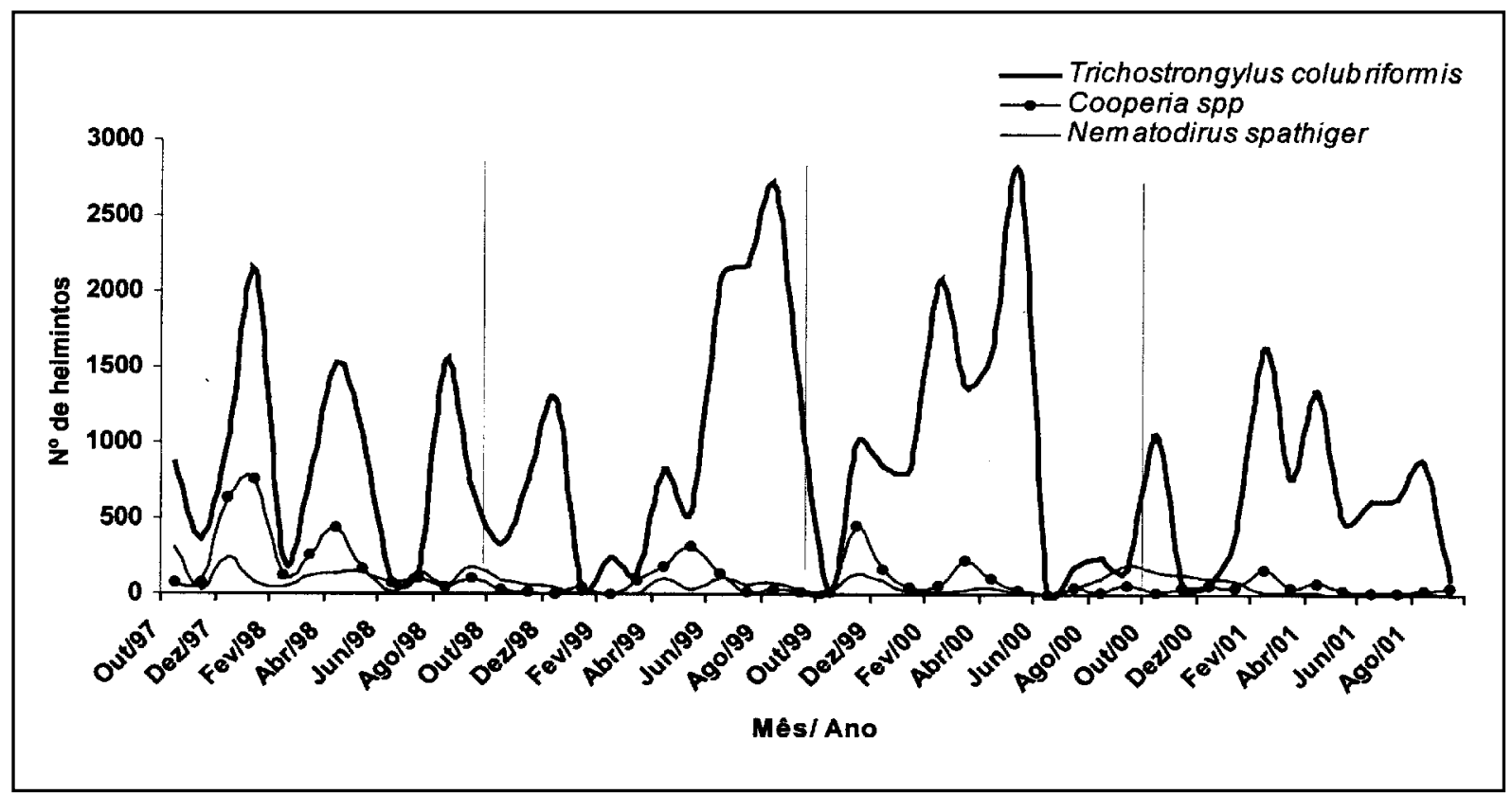

Figura 3 - Flutuação estacional de helmintos do intestino delgado de ovinos no Planalto Catarinense, no período de outubro de1997 a setembro de 2001.

venulosum foram constatadas nos exames laboratoriais realizados em Botucatu (SP) por AMARANTE (1990). As baixas infecções encontradas no presente experimento podem também estar associadas com o maior período pré-patente desta espécie e ao pequeno período que os ovinos necropsiados permaneceram à campo (28 dias).

\section{CONCLUSÕES}

A espécie Haemonchus contortus, predomina no final da primavera até o início do inverno, com maiores intensidades no verão, influenciada principalmente pelas temperaturas médias acima de $15^{\circ} \mathrm{C}$ e da precipitação pluviométrica

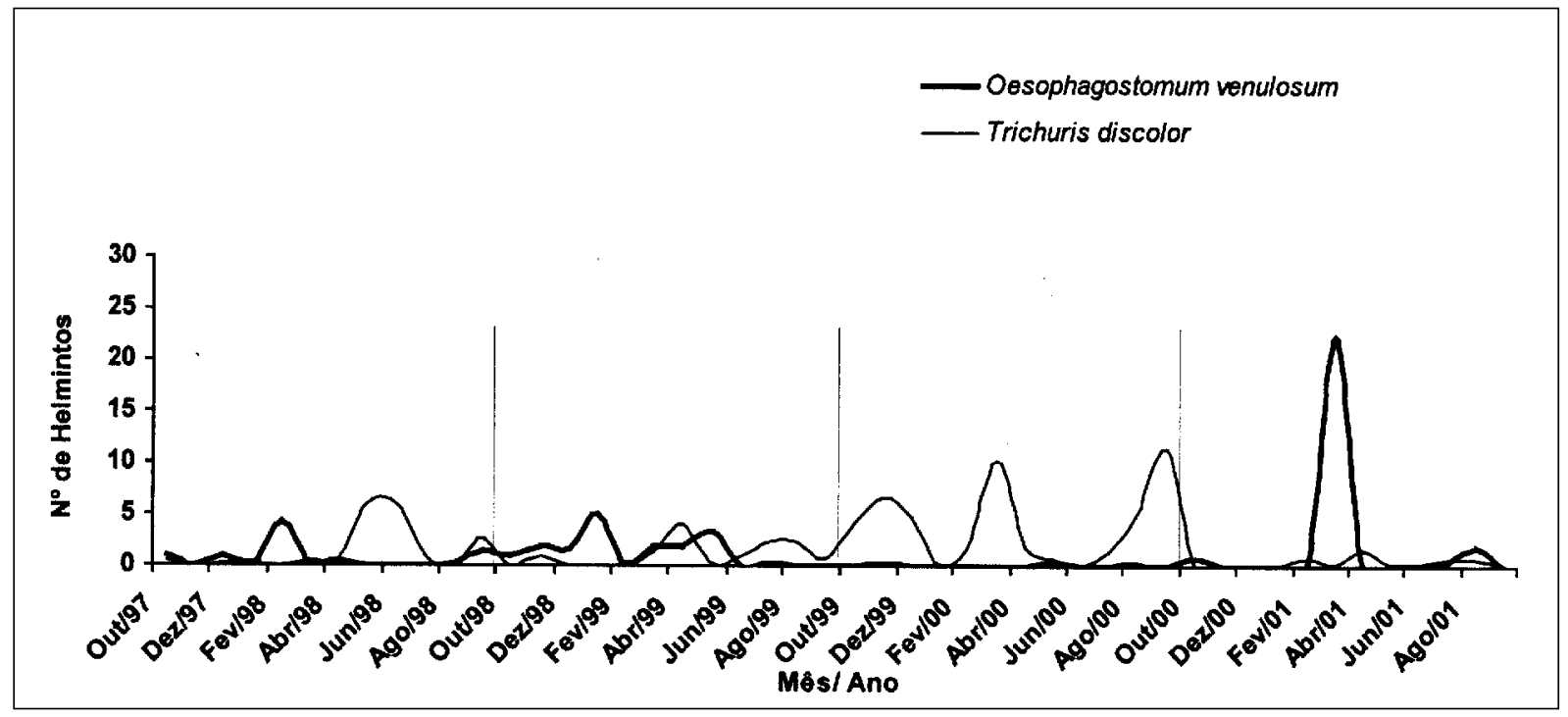

Figura 4 - Flutuação estacional de helmintos do intestino grosso de ovinos, no Planalto Catarinense, no período de outubro de1997 a setembro de 2001.

Ciência Rural, v.34, n.6, nov-dez, 2004. 
com mais de 50mm. As espécies Trichostrongylus axei e Trichostrongylus colubriformis predominam no outono até o final do inverno devido à maior adaptação às baixas temperaturas e pela menor concorrência com o Haemonchus contortus. As demais espécies presentes não preocupam com relação a medidas estratégicas de controle devido às baixas intensidades de infecção.

\section{REFERÊNCIAS BIBLIOGRÁFICAS}

AMARANTE, A.F.T. Profilaxia da verminose ovina, descontaminação de pastagens. In: CURSO DE EXTENSÃO UNIVERSITÁRIA EM PRODUÇÃO DE OVINOS, 1,2., 1989, Jaboticabal, SP. Anais... Jaboticabal : FUNEP, 1990. p.201-208.

FREITAS, M.G.; COSTA, H.M.A. Estudo da distribuição dos helmintos nas diferentes partes do sistema digestivo de bezerros. Arq Esc Vet, Belo Horizonte, v.22, p.179-196, 1970.

GONÇALVES, P.C. Epidemiologia da helmintose ovina em Guaíba (RS). 1974. 41f. Dissertação (Mestrado em Doenças Parasitárias) - Universidade Federal do Rio Grande do Sul.

GORDON, H.McL. The epidemiology of parasitic disease with special reference to studies with nematode parasite of sheep. Aust Vet J, v.24, p.17-44, 1948.

IBGE. Instututo Brasileiro de Geografia e Estatística. Censo Agropecuário, Santa Catarina, 1995 - 1996. Rio de Janeiro, 1997. 286p.

LEVINE, N.D. Weather, climate and the bionomics of ruminants. Adv Vet Sci, n.8, p.215-261, 1963.

LEVINE, N.D.;TODD, K.S.Jr; BOATMAN, P.A. Development and survival of Haemonchus contortus on pasture. Am J Vet Res, v.35, p.1412-1422, 1974.

PINHEIRO, A.da C. Aspectos da verminose dos ovinos. In: JORNADA DE PRODUÇÃO OVINA NO RS. 1., 1979, Bagé. Anais... Bagé : Empresa Brasileira de Pesquisa Agropecuária, 1979. p.139-48.

PINHEIRO, A.da C. Verminose ovina. In: CURSO SOBRE PARASITOSES DOS RUMINATES, 1., 1981, Lages, SC. Anais... Florianópolis : Colégio Brasileiro de Medicina Veterinária, 1982. p.61-75.
PINHEIRO, A.da C.; ECHEVARRIA, F.A.M.; ALVESBRANCO. F.de P.J. Epidemiologia da helmintose ovina em Bagé (RS-Brasil). In: Bagé-RS : Centro Nacional de Pesquisa em Ovinos, EMPRESA BRASILEIRA DE PESQUISA AGROPECUÁRIA, 1987. p.263-67. (Coletânea das pesquisas: Medicina Veterinária e Parasitologia. Bagé).

RAMOS, C.I.; PALOSCHI,C.G. Epidemiologia das helmintoses de bovinos de corte no Planalto Catarinense. EMPASC. Florianópolis : EMPASC, 1986. 38p. (Boletim Técnico, 37).

RAMOS, C. et al. Gastrointestinal and pulmonary helminths in sheep on the Santa Catarina Plateau. In: CONFERENCE WORLD ASSOCIATION FOR THE ADVANCEMENT OF VETERINARY PARASITOLOGY, 11., 1985, Rio de Janeiro, RJ. Resumos... Rio de Janeiro : WAAP, 1985. p.24.

REINECKE, R.K.A. An anthelmintic test for larval stages of sheep nematodes. Onderst J Vet Res, v.35, p.287-297, 1968.

REINECKE, R.K.A. Helminth diseases in domestic animals in relation to their environment. South Afr J Sci, v.66, p.92-98, 1970

RITTER, W.; SORRENSON, W.J. Produção de bovinos no Planalto de Santa Catarina - Brasil; situação atual e perspectivas. Eschborm : GTZ - Sociedade Alemã de Cooperação Técnica. 1985. 172p.

RODA, D.S et al. Noções de manejo de ovinos. Nova Odessa : Intituto de Zootecnia, 1986. p.63. (Boletim técnico, 21).

SANTIAGO, M.A.; BEVENENGA, S.F.; COSTA, U.C. Epidemiologia e controle da helmintose ovina no município de Itaqui-RS. Pesq Agrop Bras, v.11, p.1-7, 1976.

SIQUEIRA, E.R. Rev tecnologia e treinamento agropecuário. Ano 3, n.10, p.32, mar/abr, 1999.

SOULSBY, E.J.L. Parasitología y enfermidades parasitarias em los animales domésticos. 7.ed. México: Interamericana, 1987. 823p.

SOUZA, A.P. de, et al. Período para desinfestação das pastagens por larvas de nematóides gastrintestinais de ovinos, em condições naturais nos campos de Lages, SC. Rev Bras Parasitol Vet, v.9, n.2, p.159-164, 2000.

UENO, H.; GONÇALVES, P.C. Manual para diagnóstico das helmintoses de ruminantes. 4ed. Tóquio : Japan International Cooperation Agency, 1998. 145p. 